\title{
Double cnoidal waves of the Korteweg-de Vries equation: A boundary value approach
}

\author{
Sue Ellen Haupt ${ }^{1}$ and John P. Boyd \\ Department of Atmospheric, Oceanic, and Space Science, The University of Michigan, \\ 2455 Hayward Avenue, Ann Arbor, MI 48109, USA \\ Received 21 November 1988 \\ Revised manuscript received 16 June 1989 \\ Accepted 23 February 1990 \\ Communicated by R.M. Westervelt
}

\begin{abstract}
Double cnoidal waves of the Korteweg-de Vries equation are studied by direct solution of the nonlinear boundary value problem. These double cnoidal waves, which are the spatially periodic generalization of the well-known double soliton, are exact solutions with two independent phase speeds. The equation is written in terms of two phase variables and expanded in two-dimensional Fourier series. The small-amplitude solution is obtained via the Stokes' perturbation expansion. This solution is numerically extended to larger amplitude by employing a Newton-Kantorovich/continuation in amplitude/ Galerkin algorithm. The crests of the finite amplitude solution closely match the $\operatorname{sech}^{2}$ solitary wave form and the three cases of solitary wave interaction described by Lax are identified for the double cnoidal waves. This simple approach reproduces specific features such as phase shift upon collision, distinction between instantaneous and average phase speeds, and a "paradox of wavenumbers".
\end{abstract}

\section{Background}

\subsection{Polycnoidal waves}

Weakly nonlinear one-dimensional shallow water waves can be modeled with the Korteweg-de Vries (KdV) equation [1]. The KdV admits solitary wave solutions which precisely conserve energy and form upon collision. The smooth periodic generalizations, cnoidal waves, with a constant pattern of equidistant wavecrests are readily observed in channels, lakes, and oceans. However, not all wave forms have such a smooth singly periodic appearance. We often see multiple wavetrains interacting. When such wavetrains have differing wavelengths and phase speeds, the waves collide each period, causing either a single or double hump to be raised during each collision (discussed further in section 5 and demonstrated in figs. 2 through 7). Thus, the waves may appear more jumbled and time-dependent, but may often still be described by the same mathematics, merely requiring more than one wave on each period. Such patterns with multiple waves on each period, each with a distinct wavenumber and phase speed are polycnoidal or $N$-polycnoidal waves. The special case $N=1$ is the cnoidal wave. The generalized solutions $(N>1)$ are also known as finite band or finite gap solutions. When exactly two waves of differing wavenumber and phase speed interact periodically $(N=2)$, they are double cnoidal waves. For detailed reviews of polycnoidal waves see Boyd [2] or Boyd and Haupt [3].

\footnotetext{
${ }^{1}$ Present address: National Center for Atmospheric Research, P.O. Box 3000, Boulder, CO 80307, USA.
} 
How relevant are these special polycnoidal waves to the general, spatially periodic solution? The answer is that any solution may be approximated to arbitrarily high accuracy for an arbitrary, finite-time interval by an $N$-polycnoidal wave of sufficiently large $N$ and appropriately chosen parameters. Thus, to understand the polycnoidal special solutions is to understand the general solution as well.

\subsection{The KdV equation}

The Korteweg-de Vries ( $\mathrm{KdV}$ ) equation [1] is the simplest nonlinear evolution equation which admits solitary and polycnoidal wave solutions. We use the $\mathrm{KdV}$ in the form

$$
u_{t}+u u_{x}+u_{x x x}=0
$$

where the subscripts $t$ and $x$ denote differentiation in time and space respectively.

Here, we concentrate on double cnoidal waves. The solution is expressed as $u(x, t)=u(X[x, t], Y[x, t])$, where the phase variables, $X$ and $Y$, are defined as

$$
X=k_{1}\left(x-c_{1} t\right), \quad Y=k_{2}\left(x-c_{2} t\right),
$$

where $k_{1}$ and $k_{2}$ are the wavenumbers and $c_{1}$ and $c_{2}$ are the corresponding phase speeds. Since we may always rescale the period, we may take $k_{1}=1$ without loss of generality [4].

The Korteweg-de Vries equation is a good testbed for double cnoidal waves for several reasons. First, it has been rigorously proved that the KdV has double cnoidal wave solutions. Second, it is a model for various physical phenomena, including water and plasma waves, geophysical Rossby waves, and internal waves [5].

$\mathrm{N}$-polycnoidal wave solutions may be found exactly for the integrable $\mathrm{KdV}$. They were first studied using the "Hill's spectrum method," analogous to the inverse scattering method for isolated waves by Novikov [6] and Lax [7]. The technique is reviewed in detail by Dubrovin et al. [8]. In an appendix to Lax's 1975 paper [7] (by Hyman), a numerical integration indicates that the initial shape of the wave recurs, just phase-shifted in space. More recently, Osborne and Bergamasco $[9,10]$ have studied the periodic scattering problem and developed a fast numerical algorithm. An alternative approach, transforming the $\mathrm{KdV}$ into the form of the "Hirota-Korteweg-de Vries" equation which may be solved in terms of multidimensional Riemann theta functions, was discovered independently by Nakamura [11] and Boyd [4]. Boyd [12-14] uses the theta functions to further study the properties of KdV double cnoidal waves.

The two-dimensional Riemann theta functions also solve the Kandomtsev-Petviashvili (KP) equation [15], which is the two-dimensional generalization of the $\mathrm{KdV}$ and describes wavetrains interacting obliquely [16]. These waves are also double cnoidal waves since there are exactly two-phase speeds and they are described by the two-dimensional Riemann theta functions. Note that when the angle between the two wavetrains reduces to zero, the two-dimensional waves reduce to $\mathrm{KdV}$ double cnoidal waves.

We briefly review the theta function studies of Boyd [4, 12-14] so that the double cnoidal physics may be compared with the new numerical solutions computed herein. An exact solution to the $\mathrm{KdV}$ representing an $\mathrm{N}$-polycnoidal wave may be expressed as

$$
u=12[\ln \theta(X[x, t], Y[x, t])]_{x x},
$$

where $\theta$ represents an $N$-dimensional theta function. 
Boyd's theta function studies yield several important observations. First, the phase speeds $c_{1}$ and $c_{2}$ are not instantaneous phase velocities, but rather are average phase speeds. When the waves are well separated, they travel at well-defined speeds, $c_{1}^{\text {sol }}$ and $c_{2}^{\text {sol }}$, the velocities of isolated solitary waves. Upon collision, the taller wave is phase-shifted forward and the smaller backward. This process is repeated $k_{2}$ times each full period traversal. Thus, the speed of movement of each peak varies in time. The average speeds $c_{1}$ and $c_{2}$ are related to the speeds far from the wave interaction region by

$$
c_{1}=c_{1}^{\text {sol }}+\text { phase shift } / \text { time between collisions }
$$

and similarly for $c_{2}$.

Another property is the "paradox of wavenumbers" [14]. The simplest Gaussian (large-amplitude) double cnoidal wave is expressed as $k_{1}=k_{2}=1$ or $\{1,1\}$. However, a Fourier series with $k_{1}=k_{2}=1$ makes no sense, so the simplest Fourier (small-amplitude) representation is $k_{1}=1, k_{2}=2$ or [1,2]. As shown by numerical continuation [14], these two representations are equivalent.

\subsection{The boundary value approach}

The theta function representation of the $\mathrm{KdV}$ is useful for gaining insight into the properties of its solitary and polycnoidal waves; however, there are two major limitations. (1) They are only useful for studying a class of problems for which an exact solution exists. The $\mathrm{KdV}$ is exactly integrable and its solitons and polycnoidal waves preserve their form upon collision. Other evolution equations, such as the regularized long wave (RLW) equation [17] and its two-dimensional generalization [18], which equally well describe relevant waves, are not integrable. Their waves do not precisely preserve energy upon collision and they are not solvable by exact methods such as the theta function approach. (2) It would be useful to have a representation which could easily be related to observed wave forms. Hammack et al. [19] have devised a method for fitting measured data to the theta parameters for the KP equation, but the meaning of the fitted parameters are not as clear as the coefficients of a double Fourier series would be. Fourier coefficients are more useful for representing the data by standard methods, such as energy spectra.

This present work introduces a new mathematical and numerical approach which does not have these limitations. It is easily applied to nonintegrable problems [20] and could be easily applied to multidimensional equations as well. Since the Fourier coefficients are computed directly, one could easily use it to fit observed data.

This boundary value technique is demonstrated for the simplest example of polycnoidal waves - double cnoidal waves of the $\mathrm{KdV}$ equation. Our technique is to eliminate the explicit time dependence, posing a boundary value problem in two distinct phase variables, which incorporate the spatial and time dependence of the two waves. Then we solve the problem in the phase variables with periodic boundary conditions. Such a representation is simple, both conceptually and to implement, yet reproduces all of the features of the theta function studies. Boyd [21] has demonstrated that this approach gives accurate solutions for a one-dimensional problem in an easily evaluated form, yet is not difficult to use. Solutions may be found perturbatively for small amplitude waves or numerically for arbitrary amplitude. Although this technique computes an equilibrium (steadily translating) solution, it captures the full time evolution since the time is intrinsically included in the separate phase variables, each with its own phase speed. The $\mathrm{KdV}$ equation with its known solutions for comparison is the ideal testbed to demonstrate this. 
Appendix B of ref. [12] gives a Stokes' expansion for double cnoidal waves of the KdV. Here, we extend that solution to higher amplitude numerically and show that, in the large-amplitude regime, the double cnoidal waves behave as coupled solitary waves. In section 2, we first present a general Stokes perturbation expansion for double cnoidal waves of the $\mathrm{KdV}$ for arbitrary $k_{2}$. The details of the numerical Newton-Kantorovich/Galerkin algorithm are presented in section 3 and the large-amplitude results are compared to solitary waves. Then, in section 4 , we discuss how the method is useful in studying the properties of $\mathrm{KdV}$ double cnoidal waves. We investigate three separate types of interactions, note the characteristics of the waves plotted in terms of phase variables, and study double cnoidal waves with $k_{2}=3$. In section 5 , we summarize our findings and suggest directions for future study.

\section{The Stokes expansion}

In this section, we use perturbation theory to derive an analytical solution for double cnoidal waves of the $\mathrm{KdV}$. In particular, we use a singular perturbation expansion in which the phase speed as well as the independent variable is expanded as a function of an amplitude parameter. The method is described by Whitham [22] and under the name of Poincaré-Lindstedt by Nayfeh [23]. Since it was derived by Sir G.G. Stokes, we refer to it here as the Stokes expansion. To represent double cnoidal waves, we then represent the solution as a Fourier series in two phase dimensions and solve for the Fourier coefficients.

The first step in the two-dimensional Stokes expansion is to express the $\mathrm{KdV}$ (1.1) in two phase dimensions, defined here as

$$
X^{\prime}=x-c_{1} t, \quad Y^{\prime}=x-c_{2} t,
$$

where $c_{1}$ and $c_{2}$ are the phase speeds. Note that the $k_{1}$ and $k_{2}$ of (1.2) have been omitted here: $k_{1}$ is taken to be 1 and $k_{2}$ is added later as $k$ in the definition of the basis functions. Eqs. (2.1) are substituted into (1.1) and the chain rule applied to produce the two-dimensional $\mathrm{KdV}$ :

$$
\left(u-c_{1}\right) u_{X^{\prime}}+\left(u-c_{2}\right) u_{Y^{\prime}}+u_{X^{\prime} X^{\prime} X^{\prime}}+3 u_{X^{\prime} X^{\prime} Y^{\prime}}+3 u_{X^{\prime} Y^{\prime} Y^{\prime}}+u_{Y^{\prime} Y^{\prime} Y^{\prime}}=0 .
$$

The second step is to expand the function, $u$, and two phase speeds, $c_{1}$ and $c_{2}$, in powers of the amplitude parameter, $a$, which is defined to be the coefficient of $\cos \left(X^{\prime}\right)$ :

$$
\begin{aligned}
& u=a u_{1}+a^{2} u_{2}+a^{3} u_{3}+\ldots, \\
& c_{1}=c_{1,0}+a c_{1,1}+a^{2} c_{1,2}+\ldots, \\
& c_{2}=c_{2,0}+a c_{2,1}+a^{2} c_{2,2}+\ldots .
\end{aligned}
$$

Eqs. (2.3) are substituted into (2.2) and powers of $a$ are matched. The lowest-order equation is

$$
a^{1}:-c_{1,0} u_{1, X^{\prime}}-c_{2,0} u_{1, Y^{\prime}}+u_{1, X^{\prime} X^{\prime} X^{\prime}}+3 u_{1, X^{\prime} X^{\prime} Y^{\prime}}+3 u_{1, X^{\prime} Y^{\prime} Y^{\prime}}+u_{1, Y^{\prime} Y^{\prime} Y^{\prime}}=0 .
$$

We take the lowest-order solution to be

$$
u_{1}=\cos \left(X^{\prime}\right)+b \cos \left(k Y^{\prime}\right)
$$


where $k$ is an arbitrary wavenumber, and $b$ is an independent amplitude of $\mathscr{O}(1)$. Both terms are scaled by the amplitude $a$ and are the same order of magnitude, the most interesting case. Substituting (2.5) into (2.4) produces

$$
c_{1,0} \sin \left(X^{\prime}\right)+c_{2,0} b k \sin \left(k Y^{\prime}\right)+\sin \left(X^{\prime}\right)+b k^{3} \sin \left(k Y^{\prime}\right)=0
$$

from which we determine the lowest-order solution of phase speed to be

$$
c_{1,0}=-1, \quad c_{2,0}=-k^{2} \text {. }
$$

Similarly, the $i$ th order equation is

$$
\begin{aligned}
& a^{i}:-c_{1,0} u_{i, X^{\prime}}-c_{2,0} u_{i, Y^{\prime}}+u_{i, X^{\prime} X^{\prime} X^{\prime}}+3 u_{i, X^{\prime} X^{\prime} Y^{\prime}}+3 u_{i, X^{\prime} Y^{\prime} Y^{\prime}}+u_{i, Y^{\prime} Y^{\prime} Y^{\prime}}=F_{i}\left(X^{\prime}, Y^{\prime}\right), \\
& F_{i}\left(X^{\prime}, Y^{\prime}\right)=-\sum_{k=1}^{i-1}\left[\left(u_{k}-c_{1, k}\right) u_{i-k, X^{\prime}}+\left(u_{k}-c_{2, k}\right) u_{i-k, Y^{\prime}}\right] .
\end{aligned}
$$

The third step is applying a two-dimensional Fourier expansion to the solution and the right-hand side as:

$$
\begin{aligned}
& u_{i}\left(X^{\prime}, Y^{\prime}\right)=\sum_{m} \sum_{n} u_{i, m n} \cos \left(m X^{\prime}+n k Y^{\prime}\right), \\
& F_{i}\left(X^{\prime}, Y^{\prime}\right)=\sum_{m} \sum_{n} f_{i, m n} \sin \left(m X^{\prime}+n k Y^{\prime}\right),
\end{aligned}
$$

where $u_{i, m n}$ and $f_{i, m n}$ are the Fourier coefficients of the function and the right-hand side respectively. Substituting (2.8) into (2.7) produces a relationship between the function coefficients $u_{i, m n}$ and right-hand side coefficients $f_{i, m n}$ :

$$
u_{i, m n}=\frac{f_{i, m n}}{c_{1,0} m+c_{2,0} k n+m^{3}+3 m^{2} k n+3 m k^{2} n^{2}+k^{3} n^{3}} .
$$

Since the right-hand side, $F_{i}\left(X^{\prime}, Y^{\prime}\right)$, consists of algebraic combinations of solutions from lower orders, the coefficients $f_{i, m n}$ are completely specified at $i$ th order. Thus, the $u_{i, m n}$ are easily computed.

The phase speeds at $i$ th order, $c_{1, i}$ and $c_{2, i}$ are computed by requiring that the coefficients $\sin \left(X^{\prime}\right)$ and $\sin \left(k Y^{\prime}\right)$ vanish to avoid a resonance with the lowest-order solution (2.5).

For example, the right-hand side of the second-order equation is

$$
\begin{aligned}
a^{2}: F_{2}= & -c_{1,1} \sin \left(X^{\prime}\right)+\frac{1}{2} \sin \left(2 X^{\prime}\right)-b k c_{2,1} \sin \left(k Y^{\prime}\right)+\frac{1}{2} b^{2} k \sin \left(2 k Y^{\prime}\right) \\
& +\frac{1}{2} b(k+1) \sin \left(X^{\prime}+k Y^{\prime}\right)-\frac{1}{2} b(k-1) \sin \left(X^{\prime}-k Y^{\prime}\right)
\end{aligned}
$$

Eliminating the terms in $\sin \left(X^{\prime}\right)$ and $\sin \left(k Y^{\prime}\right)$ requires

$$
c_{1,1}=c_{2,1}=0 \text {. }
$$


Table 1

Fourier coefficients for the double cnoidal wave of the $\mathrm{KdV}$ equation for $k=2$ and $k=3$ as computed by the Stokes' expansion.

\begin{tabular}{llllll} 
Coefficient of & $k=2$ & $k=3$ & Coefficient of & $k=2$ & $k=3$ \\
\hline$c_{1}$ & $-1+a^{2} / 24$ & $-1+a^{2} / 24$ & $\cos (3 k Y)$ & $a^{3} b^{3} / 3072$ & $a^{3} b^{3} / 15552$ \\
$c_{2}$ & $-4+a^{2} b^{2} / 96$ & $-9+a^{2} b^{2} / 216$ & $\cos (X+k Y)$ & $a^{2} b / 12$ & $a^{2} b / 18$ \\
$\cos (X)$ & $a$ & $a$ & $\cos (X-k Y)$ & $-a^{2} b / 12$ & $-a^{2} b / 18$ \\
$\cos (k Y)$ & $a b$ & $a b$ & $\cos (X+2 k Y)$ & $25 a^{3} b^{2} / 10368$ & $25 a^{3} b^{2} / 62208$ \\
$\cos (2 X)$ & $a^{2} / 12$ & $a^{2} / 12$ & $\cos (X-2 k Y)$ & $-a^{3} b^{2} / 128$ & $-25 a^{3} b^{2} / 15552$ \\
$\cos (3 X)$ & $a^{3} / 192$ & $a^{3} / 192$ & $\cos (2 X+k Y)$ & $a^{3} b / 162$ & $25 a^{3} b / 6912$ \\
$\cos (2 k Y)$ & $a^{2} b^{2} / 48$ & $a^{2} b^{2} / 108$ & $\cos (2 X-k Y)$ & 0 & $-a^{3} b / 11728$
\end{tabular}

The remaining terms show that $f_{2,20}=\frac{1}{2}, f_{2,02}=\frac{1}{2} b^{2} k, f_{2,11}=\frac{1}{2} b(k+1)$, and $f_{2,1-1}=-\frac{1}{2} b(k-1)$. Therefore, by (2.9), $u_{2,20}=\frac{1}{12}, u_{2,12}=b^{2} / 12 k^{2}, u_{2,11}=b / 6 k, u_{2,1-1}=-b / 6 k$. Thus, the second-order solution is

$$
u_{2}=\frac{1}{12} \cos \left(2 X^{\prime}\right)+\frac{b^{2}}{12 k^{2}} \cos \left(2 k Y^{\prime}\right)+\frac{b}{6 k} \cos \left(X^{\prime}+k Y^{\prime}\right)-\frac{b}{6 k} \cos \left(X^{\prime}-k Y^{\prime}\right)
$$

Similarly, at third order we find

$$
c_{1,2}=1 / 24, \quad c_{2,2}=b^{2} / 24 k^{2} .
$$

The solution for $u_{3}$ contains terms in $\cos \left(3 X^{\prime}\right), \cos \left(3 k Y^{\prime}\right), \cos \left(X^{\prime}+2 k Y^{\prime}\right), \cos \left(X^{\prime}-2 k Y^{\prime}\right), \cos \left(2 X^{\prime}+k Y^{\prime}\right)$, and $\cos \left(2 X^{\prime}-k Y^{\prime}\right)$. Table 1 lists the phase speeds and coefficients to third order for the cases $k=2$ and $k=3$. The algebraic computations were accomplished using the REDUCE algebraic manipulation language.

Before leaving the topic of the Stokes' expansion we must note that when the denominator of (2.9) vanishes we have harmonic resonance. The lowest $\mathrm{KdV}$ resonance is $m=5, n=-4(k=2)$. This mode would be expected to appear at ninth order in the perturbation expansion (well beyond the results of table 1); however, when computed numerically, it actually was $\mathscr{O}\left(a^{7}\right)$. Haupt and Boyd [24] explain how to overcome this difficulty. There, the resonant wavenumber is added in at the order of resonance with an unknown coefficient to be determined at higher order. If one required a perturbation solution accurate to $a^{7}$, the $(5,-4)$ wave would necessarily be added at seventh order, but the computation of its coefficient would be delayed until ninth order. Our purpose here is to use the KdV Stokes double cnoidal wave expansion as a starting point for a numerical solution; thus, such a high-order expansion is not required. Our previous paper, however, shows how the series can be extended to as high an order as we wish despite the resonances.

\section{Numerical solution}

The perturbation method of the previous section is useful only for small-amplitude waves. Here, we demonstrate a numerical technique for extending the Stokes series to larger amplitude. The numerical method is a combination of the Newton-Kantorovich technique with continuation in amplitude and Fourier Galerkin discretization. 
In the Newton-Kantorovich method, the iteration is applied directly to the differential equation, then the linearized equation is discretized-here, spectrally using two-dimensional Fourier series. This iterative method was presented by Kantorovich [25], described by Norton [26], and applied to one-dimensional nonlinear wave problems by Boyd [21]. Specifically, we approximate the function and two phase speeds as current iterates, or loosely, "guesses" plus corrections:

$$
u=u^{i}+\Delta, \quad c_{1}=c_{1}^{i}+\delta_{c_{1}}, \quad c_{2}=c_{2}^{i}+\delta_{c_{2}},
$$

where the superscript " $i$ " denotes the result of the previous iteration (or first guess if $i=0$ ) and the $\Delta$ and $\delta$ 's represent the corrections to be computed. Substituting (3.1) into (2.2) and linearizing in the corrections produces the operator equation:

$$
L \Delta+l_{1} \delta_{c_{1}}+l_{2} \delta_{c_{2}}=f
$$

where

$$
\begin{aligned}
& L=\left(u^{i}-c_{1}^{i}\right) \frac{\partial}{\partial X^{\prime}}+\left(u^{i}-c_{2}^{i}\right) \frac{\partial}{\partial Y^{\prime}}+u_{X^{\prime}}^{i}+u_{Y^{\prime}}^{i}+\frac{\partial^{3}}{\partial X^{\prime 3}}+3 \frac{\partial^{3}}{\partial X^{\prime 2} \partial Y^{\prime}}+3 \frac{\partial^{3}}{\partial X^{\prime} \partial Y^{\prime 2}}+\frac{\partial^{3}}{\partial Y^{\prime 3}}, \\
& l_{1}=-u_{X^{\prime}}^{i}, \quad l_{2}=-u_{Y^{\prime}}^{i}, \\
& f\left(X^{\prime}, Y^{\prime}\right)=-\left[\left(u^{i}-c_{1}^{i}\right) u_{X^{\prime}}^{i}+\left(u^{i}-c_{2}^{i}\right) u_{Y^{\prime}}^{i}+u_{X^{\prime} X^{\prime} X^{\prime}}^{i}+3 u_{X^{\prime} X^{\prime} Y^{\prime}}^{i}+3 u_{X^{\prime} Y^{\prime} Y^{\prime}}^{i}+u_{Y^{\prime} Y^{\prime} Y^{\prime}}^{i}\right] .
\end{aligned}
$$

The lowest-order solution is taken to be (2.5). The right-hand side of (3.2) is completely determined by the previous iterate, so we are left with a linearized system to solve for the corrections to the coefficients, $\delta_{m n}$, and to the phase speeds, $\delta_{c_{1}}$ and $\delta_{c_{2}}$.

To discretize (3.2), we first expand the solution and the correction as two-dimensional Fourier series:

$$
\begin{aligned}
& u\left(X^{\prime}, Y^{\prime}\right) \simeq u_{M N}\left(X^{\prime}, Y^{\prime}\right)=\sum_{m=0}^{M} \sum_{n=-N}^{N} u_{m n} \cos \left(m X^{\prime}+n k Y^{\prime}\right), \\
& \Delta=\sum_{m=0}^{M} \sum_{n=-N}^{N} \delta_{m n} \cos \left(m X^{\prime}+n k Y^{\prime}\right) .
\end{aligned}
$$

The discretized Newton-Kantorovich operator equation is then solved using a Galerkin method. In computing the Galerkin matrix, we take the inner product of (3.2) with an orthogonal basis function, $\sin \left(m_{i} X^{\prime}+n_{i} k Y^{\prime}\right)$. The constant coefficient terms produce zero elements everywhere except along the diagonal. Nonzero values elsewhere are from the terms composed of products of the previous iterate with the current Fourier expansion. Thus, the Galerkin matrix is asymptotically diagonal and well-conditioned. The matrix equation is of the form

$$
\mathbf{H} \boldsymbol{\delta}+\mathbf{h}_{1} \boldsymbol{\delta}_{c_{1}}+\mathbf{h}_{2} \boldsymbol{\delta}_{c_{2}}=\boldsymbol{g}
$$


where

$$
\begin{aligned}
& H_{i j}=\left(\sin \left(m_{i} X^{\prime}+n_{i} k Y^{\prime}\right), L \cos \left(m_{j} X^{\prime}+n_{j} k Y^{\prime}\right)\right), \\
& h_{1 i}=\left(\sin \left(m_{i} X^{\prime}+n_{i} k Y^{\prime}\right),-u_{X^{\prime}}^{i}\right), \\
& h_{2 i}=\left(\sin \left(m_{i} X^{\prime}+n_{i} k Y^{\prime}\right),-u_{Y^{\prime}}^{i}\right), \\
& g_{i}=\left(\sin \left(m_{i} X^{\prime}+n_{i} k Y^{\prime}\right), f\left(X^{\prime}, Y^{\prime}\right)\right) .
\end{aligned}
$$

The outer parentheses denote inner products $\left((u, v)=\int_{-\pi}^{\pi} \int_{-\pi}^{\pi} u\left(X^{\prime}, Y^{\prime}\right) v\left(X^{\prime}, Y^{\prime}\right) \mathrm{d} X^{\prime} \mathrm{d} Y^{\prime}\right)$. The operator, $L$, and the right-hand side, $f\left(X^{\prime}, Y^{\prime}\right)$, are defined as in (3.2).

In matrix form, the equation is

$$
\left(\begin{array}{cccccc}
H_{11} & H_{12} & \cdots & H_{1 \bar{N}} & h_{11} & h_{21} \\
H_{21} & H_{22} & \cdots & H_{2 \tilde{N}} & h_{12} & h_{22} \\
\vdots & \vdots & \ddots & \vdots & \vdots & \vdots \\
H_{\bar{N} 1} & H_{\bar{N} 2} & \cdots & H_{\bar{N} \tilde{N}} & h_{1 \bar{N}} & h_{2 \bar{N}}
\end{array}\right)\left(\begin{array}{c}
\delta_{1} \\
\delta_{11} \\
\vdots \\
\delta_{\bar{N}-2} \\
\delta_{c_{1}} \\
\delta_{c_{2}}
\end{array}\right)=\left(\begin{array}{c}
g_{1} \\
g_{2} \\
g_{3} \\
\vdots \\
g_{\bar{N}-1} \\
g_{\tilde{N}}
\end{array}\right) .
$$

$\tilde{N}$ is the total number of basis functions and the roman subscripts on the $\delta$ 's indicate the double indexing of (3.4). Although the matrix includes two additional rows to solve for the corrections to the phase speeds, it is still a square matrix with dimension $\tilde{N}$. Those two rows replace the two which would otherwise specify the coefficients of $\cos \left(X^{\prime}\right)$ and $\cos \left(k Y^{\prime}\right)$; that is, $a$ and $b$ are fixed parameters and we do not have to include them in the unknowns.

The remaining problem is to compute the inner products in (3.5). Numerical integration is not necessary since the operator, $L$, applied to $\cos \left(m_{j} X^{\prime}+n_{j} k Y^{\prime}\right)$ as well as the $-u_{X^{\prime}}^{0},-u_{Y^{\prime}}^{0}$, and $f\left(X^{\prime}, Y^{\prime}\right)$ are expressed in a Fourier representation. The nonlinear terms of $L \cos \left(m_{j} X^{\prime}+n_{j} k Y^{\prime}\right)$ and $f\left(X^{\prime}, Y^{\prime}\right)$ require a bit more attention. However, the product of two trigonometric functions may always be reduced to a sum of two trigonometric functions by applying the appropriate identities. The inner products may be analytically evaluated using the orthogonality of the trigonometric functions.

\subsection{Some program details}

The basis set, and thus, matrix dimension may be reduced by the following. First, (2.2) possesses $\mathrm{C}_{2}$ symmetry, that is, a transformation of $\left(X^{\prime}, Y^{\prime}\right) \rightarrow\left(-X^{\prime},-Y^{\prime}\right)$ is an identity transformation. Thus only cosine basis functions in the form $\cos \left(m X^{\prime}+n k Y^{\prime}\right)$ are necessary. In addition, since the cosine function is even, the terms with $m=0$ and $n<0$ may be incorporated into $\cos \left(-n k Y^{\prime}\right.$ ) (where $\left.-n>0\right)$. We also do not need terms with $(m, n)=(1,0),(0,1)$, and $(0,0)$ since $u_{10}=a, u_{01}=a b$, and $\int_{-\pi}^{\pi} u(x, t) \mathrm{d} x=0$. However, we require two additional degrees of freedom to solve for the unknown phase speeds, $c_{1}$ and $c_{2}$, so that the total number of basis functions required for rectangular truncation, that is, $N=M=k_{\max }$, is

$$
\tilde{N}_{\text {rectangular }}=2\left(k_{\max }^{2}+k_{\max }\right) .
$$


However, the magnitude of the coefficients drops off rapidly with $\sqrt{m^{2}+n^{2}}$. Therefore, it is more efficient to use a circular truncation (as shown by Orszag [27]) in which a maximum wavenumber, $k_{\max }$, is determined and all terms for which $\sqrt{m^{2}+n^{2}}>k_{\max }$ are neglected. The total number of basis functions is then

$$
\tilde{N}_{\text {circular }}=2 \sum_{i=0}^{k_{\max }-1} \operatorname{INT}\left(\sqrt{k_{\max }^{2}-i^{2}}\right)
$$

where the function INT denotes truncation to the next lowest integer. This produces considerable savings. For $k_{\max }=3, \tilde{N}_{\text {rectangular }}=24$ while $\tilde{N}_{\text {circular }}=14$. For $k_{\max }=12, \tilde{N}_{\text {rectangular }}=312$ while $\tilde{N}_{\text {circular }}=$ 220 , saving 92 degrees of freedom and considerable computer time.

The computations are done in a series of three iterative levels. The inner iteration is the Newton-Kantorovich/Galerkin iteration. Standard NAAS library routines are used to solve the matrix equation via $L U$ decomposition and back substitution. The iteration continues until the normalized root mean square error, RMSE, (computed as RMSE $\left.=\left[\left(\delta_{c_{1}}^{2}+\delta_{c_{2}}^{2}+\sum_{i=1}^{\bar{N}-2} \delta_{i}^{2}\right) /\left(c_{1}^{2}+c_{2}^{2}+\sum_{i=1}^{\bar{N}-2} u_{i}^{2}\right)\right]^{1 / 2}\right)$ is less than $10^{-8}$. Since the Jacobian matrix changes little from iteration to iteration and the LU decomposition is the rate-determining step $\left(\mathscr{O}\left(\tilde{N}^{3}\right)\right)$, it is not updated and decomposed at each iteration. Sensitivity studies to updating the matrix showed that, for moderate amplitudes, the number of iterations required for convergence changed little when not updating the Jacobian, although CPU time did decrease.

The second loop is in $k_{\max }$. The purpose of this iteration is twofold. First, only the required number of degrees of freedom to reach a chosen error bound are used. Second, the results from the previous value of $k_{\max }$ provide a good first guess for the current $k_{\max }$. For example, the problem is first solved for $k_{\max }=2$ (6 degrees of freedom), and then for $k_{\max }=3$ (14 degrees of freedom). If the change in computed coefficients and phase speeds is sufficiently small, the iteration in $k_{\max }$ is stopped. If not, we continue to higher $k_{\max }$. The criteria for stopping the iteration in $k_{\max }$ is based on the absolute changes in the four largest coefficients $\left(u_{02}, u_{20}, u_{11}\right.$, and $\left.u_{1-1}\right)$ plus the two phase speeds. In addition, the residual is computed for each value of $k_{\max }$ (by summing the solution at various values of $\left(X^{\prime}, Y^{\prime}\right)$ and substituting back into eq. (2.2)). We find that the residual may be made arbitrarily small by using a large enough $k_{\max }$; however, defining a stopping criterium based on changes in the coefficients conserves the required degrees of freedom, yet assures that the coefficients are accurate; for instance, see table 2 , which compares the maximum computed residual for various values of $k_{\max }$ for the case where $a=5$, $b=1$. Also included are the phase speeds and four largest coefficients. We note that, although a $k_{\max }$ of 13 was required for the maximum residual to drop below $1 \times 10^{-3}$, the phase speeds and largest coefficients changed negligibly beyond $k_{\max }=6$. Since we are interested in using the coefficients to produce values of the function to display graphically, the additional degrees of freedom are not needed. One would expect the residual to be an overly stringent criterion, since it contains third derivatives. The coefficients of the series for the third derivatives is that of the undifferentiated coefficients multiplied by the cube of the wavenumber. For large wavenumber and large enough amplitude for the coefficient to be significant, the third derivative terms contribute to a large residual.

The third, or outer, iteration is in amplitude, a. For small a computations, this iteration is not necessary; the perturbative first guess is sufficient for convergence. At higher amplitude, a better first guess is obtained by linearly extrapolating from the solutions at the two previous values of $a$. The continuation proceeds using a lower resolution (smaller $k_{\max }$ ) than required for the final amplitude. This is analogous to the technique of Bank and Chan [28], who showed it is sufficient to use fewer degrees of 
Table 2

Comparison of maximum residuals, phase speeds, and largest coefficients for various values of $k_{\max }$ (and thus basis functions) for $a=5, b=1$.

\begin{tabular}{cccccccc}
\hline$k_{\text {max }}\left(N_{\text {bas }}\right)$ & Resid $_{\max }$ & \multicolumn{1}{c}{$c_{1}$} & $c_{2}$ & $u_{02}$ & $u_{11}$ & $u_{01}$ & $u_{20}$ \\
\hline $2(6)$ & $6 \times 10^{1}$ & -0.56527 & -4.01854 & 0.364 & -2.15 & 1.75 \\
$3(14)$ & $3 \times 10^{1}$ & -0.01566 & -3.59455 & 0.246 & -1.98 & 1.92 & 1.27 \\
$4(24)$ & $2 \times 10^{1}$ & 0.10533 & -3.53719 & 0.239 & -1.96 & 1.93 & 1.85 \\
$5(40)$ & $7 \times 10^{0}$ & 0.12594 & -3.53141 & 0.238 & -1.96 & 1.93 & 1.87 \\
$6(56)$ & $2 \times 10^{0}$ & 0.12878 & -3.53090 & 0.238 & -1.96 & 1.93 & 1.87 \\
$7(74)$ & $9 \times 10^{-1}$ & 0.12914 & -3.53086 & 0.238 & -1.96 & 1.93 & 1.87 \\
$8(98)$ & $3 \times 10^{-1}$ & 0.12919 & -3.53086 & 0.238 & -1.96 & 1.93 & 1.87 \\
$9(126)$ & $9 \times 10^{-2}$ & 0.12919 & -3.53086 & 0.238 & -1.96 & 1.93 & 1.87 \\
$10(158)$ & $3 \times 10^{-2}$ & 0.12919 & -3.53086 & 0.238 & -1.96 & 1.93 & 1.87 \\
$11(188)$ & $9 \times 10^{-3}$ & 0.12919 & -3.53086 & 0.238 & -1.96 & 1.93 & 1.87 \\
$12(220)$ & $2 \times 10^{-3}$ & 0.12919 & -3.53086 & 0.238 & -1.96 & 1.93 & 1.87 \\
$13(264)$ & $8 \times 10^{-4}$ & 0.12919 & -3.53086 & 0.238 & -1.96 & 1.93 & 1.87 \\
\hline
\end{tabular}

freedom for the continuation steps, then to increase degrees of freedom (and thus, accuracy) at chosen "target points". As applied here, the initial amplitude solution is found using the $k_{\max }$ necessary to satisfy the chosen stopping criteria, then the iteration in amplitude is continued without increasing the number of degrees of freedom. When the final, or target amplitude is reached, iteration in $k_{\max }$ is again done to reach the desired resolution at the larger amplitude.

\subsection{Validation of numerical method}

The Newton-Kantorovich scheme for computing double cnoidal waves of the $\mathrm{KdV}$ is validated on three levels: first, we show that it produces correct results; second, we demonstrate that we are in an amplitude regime that differs from perturbation theory; and third, we show that the large-amplitude solution resembles solitary waves.

The first level is easily addressed by referring again to table 2 . The acid test of any numerical solution to a differential equation is to plug the solution back into the equation. A small residual indicates a correct solution. In this case, the residual was computed at 25 points and the maximum value chosen for table 2 . As seen there, the residual may be made arbitrarily small by using enough basis functions. (However, as discussed previously, a small residual is not essential to a sufficiently accurate solution.)

The second level of validation requires showing that we are studying an amplitude regime which is highly nonlinear. The algorithm works well beyond the amplitude where the Stokes perturbation theory provides a good solution. Table 3 lists the normalized absolute differences between the numerical and the perturbation values of the phase speeds, one of the largest coefficients $\left(u_{02}\right)$, and the sum of the absolute differences for all coefficients plus phase speeds for four values of amplitude. For an amplitude of 0.1 , the differences are tiny; they are still rather small for $a=1$. However, for $a=5$, and particularly for $a=10$, the numerical solution differs widely from the perturbation theory. At $a=10$, the perturbation computation of $c_{2}$ is in error by $557 \%$ and the absolute sum of the differences in coefficients and phase speeds is 41.1 . Therefore, since perturbation theory fails miserably for $a=10$, this case is highly nonlinear.

The third level of validation consists of comparing the numerical solution to the exact solitary wave solution in the large-amplitude limit. Although the exact double cnoidal wave solution is known (in terms of theta functions), it is not easily computed. In addition, the periodic elliptic solution (in terms of $c n$ ) is 
Table 3

Difference between numerical solution and perturbation theory of the $\mathrm{KdV}(b=1)$.

\begin{tabular}{rllll}
\multicolumn{1}{c}{$a$} & $\Delta c_{1}{ }^{\mathrm{a})}$ & $\Delta c_{2}{ }^{\mathrm{b})}$ & $\Delta u_{02}{ }^{\mathrm{c}}$ & $\Sigma^{\mathrm{d})}$ \\
\hline 0.1 & $1.69 \times 10^{-8}$ & $9.67 \times 10^{-9}$ & $2.49 \times 10^{-4}$ & $2.67 \times 10^{-7}$ \\
1.0 & $1.75 \times 10^{-4}$ & $9.63 \times 10^{-5}$ & $2.54 \times 10^{-1}$ & $4.05 \times 10^{-3}$ \\
5.0 & 0.677 & $5.91 \times 10^{-2}$ & 1.19 & 2.82 \\
10.0 & 0.243 & 5.57 & 3.38 & 41.1 \\
\hline
\end{tabular}

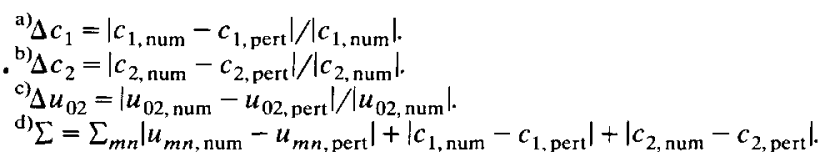

not useful since nonlinearity precludes superposition. Instead, we choose an amplitude regime for which two solitary waves per period is a good approximation. To do this, the waves must be tall enough and narrow enough so that they overlap very little with the waves on the next period. We choose to do this at the time of least-interaction ( $t=0$ in the numerical calculation) which conservatively models the overlap since the distance to the waves on the adjoining period interval is minimized. That is, two solitons on a period of length $2 \pi$ are separated a distance of $\pi$ at the time of least-interaction; however, during the interaction, the interacting peaks are a distance $2 \pi$ from the next interacting set. Thus, the shorter separation at least-interaction is more conservative for comparing the overlapping solitons. We fit the double cnoidal wave by $u_{\text {sol }}$ defined by

$$
u_{\mathrm{sol}}=\sum_{i=-2}^{2} 3 a_{1} \operatorname{sech}^{2}\left[\frac{1}{2} \sqrt{a_{1}}(x-2 \mathrm{i} \pi)\right]+\sum_{i=-2}^{2} 3 a_{2} \operatorname{sech}^{2}\left[\frac{1}{2} \sqrt{a_{2}}(x-(2 i+1) \pi)\right]+\alpha
$$

The two $\operatorname{sech}^{2}$ 's represent solitons of different amplitudes. The constant, $\alpha$ is necessary to redefine the orientation of the $x$-axis (since $\int_{-\pi}^{\pi} u(x, t) \mathrm{d} x=0$ ). The constants $a_{1}, a_{2}$, and $\alpha$ are fit numerically for best agreement with the Newton-Kantorovich solution. A quick glance at the numerical values of the wave peaks at 0 and $\pi$ and trough at $\pi / 2$ provide a good first guess. The constants are then adjusted using an iterative search in order to minimize the square of the error from the numerical solution. The results are shown in fig. 1 for $a=10$ with $b=1$. The "exact" soliton solution is indistinguishable from the Newton-Kantorovich solution. Not only does this demonstrate that this amplitude is far into the soliton regime, but it also reassures us that the solution is computed correctly.

\section{Results}

\subsection{Time evolution}

To compute $u(x, t)$ from the solution of the boundary value problem, one must evaluate $X(x, t)$ and $Y(x, t)$ from their definitions (eqs. (1.2)) and then sum the Fourier series in $X$ and $Y$. Thus we may observe the time evolution of the double cnoidal wave, and in particular, the interaction upon collision of the two wave crests. Lax [29] distinguishes three cases of solitary wave interaction, depending on the ratio of the amplitudes of the two solitons. Here, we are able to identify the same three regimes for the interaction of the two crests of the double cnoidal wave. The amplitudes of our numerical solution which 


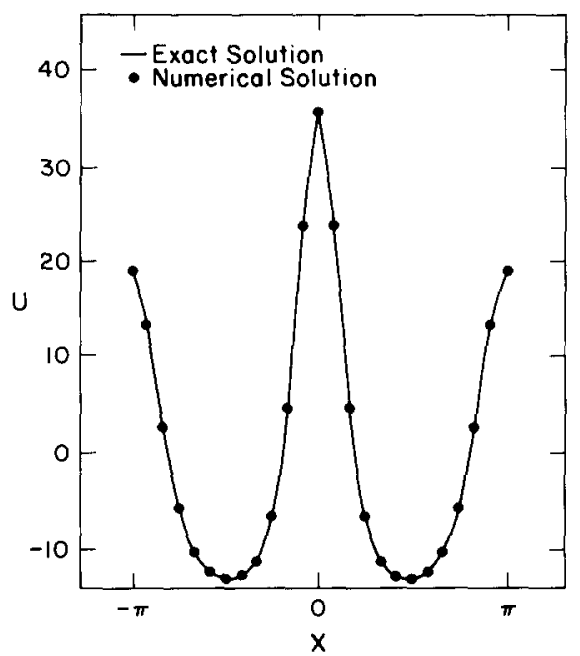

Fig. 1. Comparison of computed cnoidal wave of the $\mathrm{KdV}$ to superimposed solitary waves (the "exact" solution) for $a=10$, $b=1, t=0$.

correspond to those of Lax are those of the fitted pair of solitary waves described by (3.9). The three cases are as follows:

Case 1. This is the interaction of two similar-sized solitary waves with $a_{1} / a_{2}<2.618$. The larger wave overtakes the smaller, losing height upon approach while the smaller wave grows. They interchange roles without passing through one another, but experiencing phase shifts. This case is shown for numerically computed double cnoidal waves in figs. 2 and 3 for $a=10, b=1\left(a_{1}=16.665, a_{2}=11.126, a_{1} / a_{2}=1.498\right)$. The $x$ variable is adjusted so that the central wave is stationary in a reference frame moving with speed $c_{1}$, thus transforming the abscissa variable to the phase variable $X$. (This moving reference frame will be used in all the $x-t$ plots in this section.) The time variable runs from $t=0$ to a full recurrence time,

$$
t_{\mathrm{rec}}=2 \pi / k_{2}\left(c_{1}-c_{2}\right)
$$

The waves' evolution is depicted in two ways. Fig. 2 shows the waves' shapes as computed numerically at three different times: $t=0$ (solid lines), $t=\frac{1}{4} t_{\mathrm{rec}}$ (long dashes), and $t=\frac{1}{2} t_{\mathrm{rec}}$ (short dashes). Since the double cnoidal wave is simply periodic in time in the reference frame moving with speed $c_{1}$, the shape at $t=\frac{3}{4} t_{\mathrm{rec}}$ is the reflection of the $\frac{1}{4} t_{\mathrm{rec}}$ curve. Fig. 3 is a contour plot showing the continuous evolution over a full recurrence period, with $X$ as the abscissa and $t$ as the ordinate. At $t=0$, the wave crests are visible at the center and at the extremes of the spatial period, with troughs between. As the waves approach, the large wave shrinks as the small one grows. At all times, there are two distinct waves, even at the time of collision $\left(t=\frac{1}{2} t_{\mathrm{rec}}\right)$ as evident in fig. 2. This fact is further manifested in the contour plot (fig. 3) as the contours between the crests and troughs stretch from $t=0$ to the full recurrence time unbroken.

Case 2. Lax showed that when the amplitude of two solitary waves are sufficiently different $\left(a_{1} / a_{2}>3\right)$, the larger wave absorbs the smaller upon collision, then reemits it. Figs. 4 and 5 illustrate this for a double cnoidal wave with $a=10, b=0.25\left(a_{1}=11.828, a_{2}=2.735, a_{1} / a_{2}=4.325\right)$. Fig. 4 indicates the single hump at the time of collision $\left(t=\frac{1}{2} t_{\text {rec }}\right.$ ). The contour plot (fig. 5) shows the contrast with case 1 


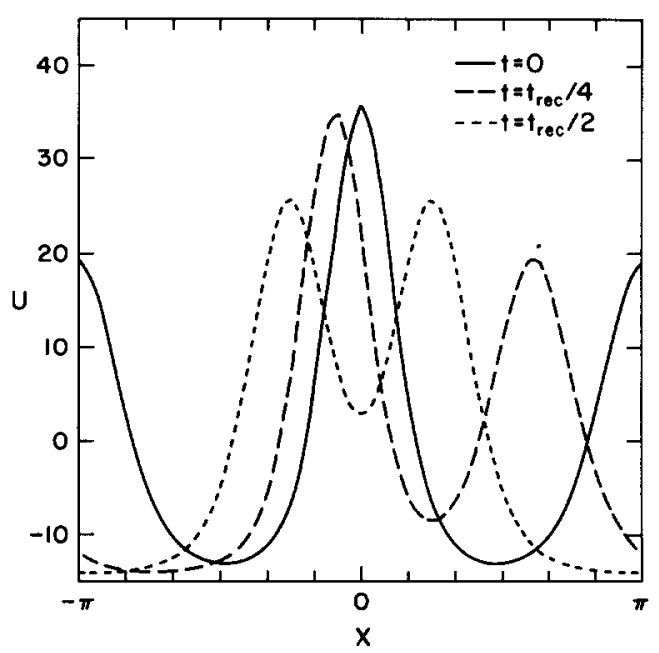

Fig. 2. Time evolution of the $\mathrm{KdV}$ double cnoidal wave for $a=10, b=1$.

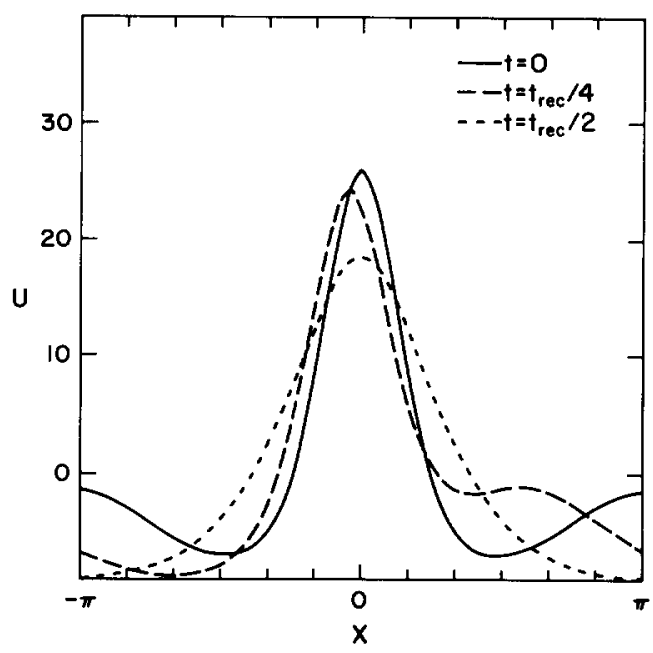

Fig. 4. Time evolution of the $\mathrm{KdV}$ double cnoidal wave for $a=10, b=0.25$.

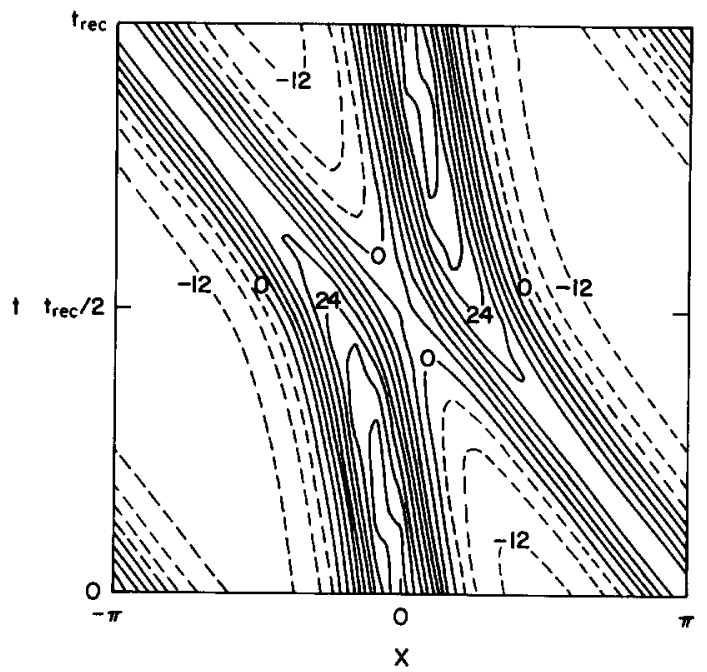

Fig. 3. Contour of time evolution for $a=10, b=1$. This is the first case of Lax, where the two waves are distinct at all times.

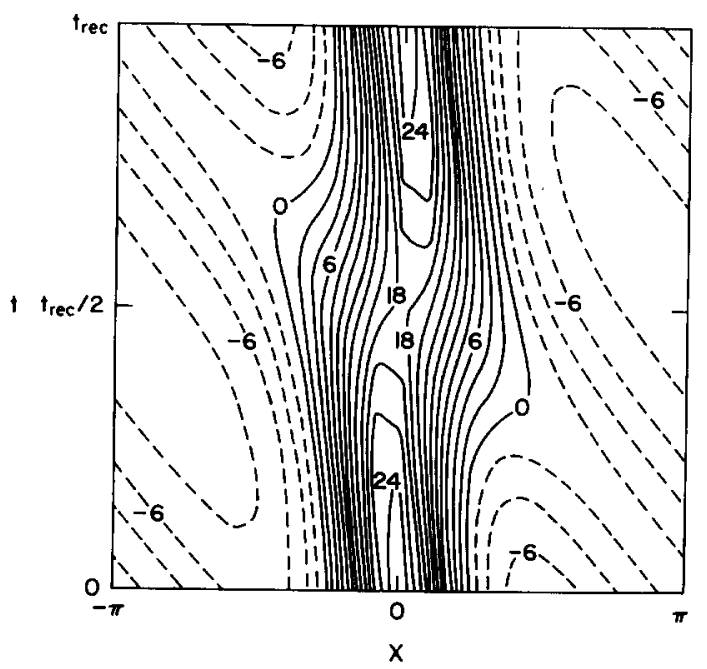

Fig. 5. Contour of time evolution for $a=10, b=0.25$. This is the second case of Lax, where the smaller wave is absorbed and reemitted. 


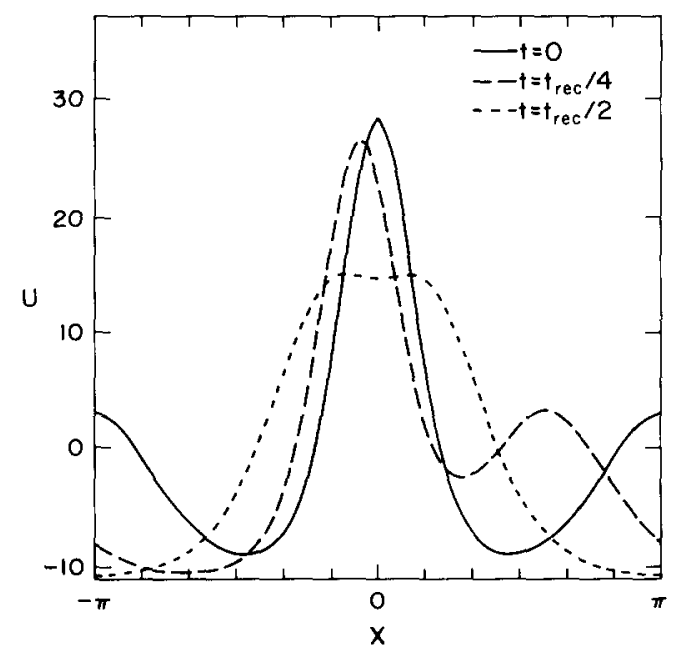

Fig. 6. Time evolution of the KdV cnoidal wave for $a=10$, $b=0.45$.

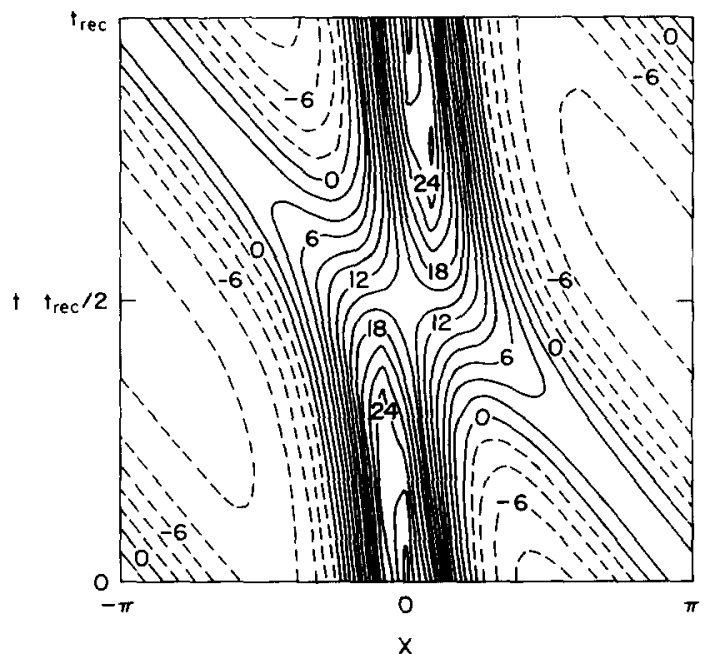

Fig. 7. Contour of $\mathrm{KdV}$ double cnoidal wave for $a=10$, $b=0.45$. This is the third case of Lax, where the waves merge but always have two humps.

(fig. 3). The center of the plot, which depicts the interaction, shows the merging of the two waves: the entire center is encompassed by a large broad peak and the trough lines do not pass through the center.

Case 3. The final case is the intermediate one where $2.618<a_{1} / a_{2}<3$ and is shown in figs. 6 and 7 for $a=10, b=0.45\left(a_{1}=14.148, a_{2}=4.738, a_{1} / a_{2}=2.775\right)$. At the time of intersection, the large wide hump has two small peaks (fig. 6). In fig. 7 , like fig. 5, the trough lines do not pass through the center. However, there is no period of time without two distinct peaks. Lax describes this case as the larger wave absorbing the smaller, raising a secondary peak, then reemitting it.

Thus, we see that the regimes of solitary wave collisions are evident in the interaction of the double cnoidal wave crests. This is not particularly surprising since double cnoidal waves approach a double soliton solution for sufficiently large amplitude.

\subsection{Phase variable analysis}

Additional insight may be gained by eliminating the time dependence and analyzing the behavior in the phase variables, $X=x-c_{1} t$ and $Y=k_{2}\left(x-c_{2} t\right)$ where we have explicitly incorporated the $k_{2}$ into the phase variable $\left(k_{1}=1\right)$. The near-linear (small-amplitude) solution is $u(X, Y) \simeq a \cos (X)+a b \cos (Y)$; therefore, the function is sinusoidal in each variable and a plot of $u$ in the $X-Y$ plane is similar to one of intersecting sine waves. This is indeed evident in the series of phase variable plots, figs. 8-12. The first, fig. 8 , is a three-dimensional surface relief plot for a basic case $(a=5, b=1)$. Along the plot edges in both the $X$ and the $Y$ direction lie the basic cosine-like cnoidal wave forms. The center hump depicts the interaction. The remaining figures are contour plots on the $X-Y$ plane.

Figs. 9 and 10, which have $a=1$ and $a=10$ respectively with $b$ fixed at 1 , illustrate several effects of varying amplitude. First, the small-amplitude plot has a maximum peak upon collision of roughly the sum of the two linear cosine waves. As $a$ increases, the maximum height in the phase space differs more from 


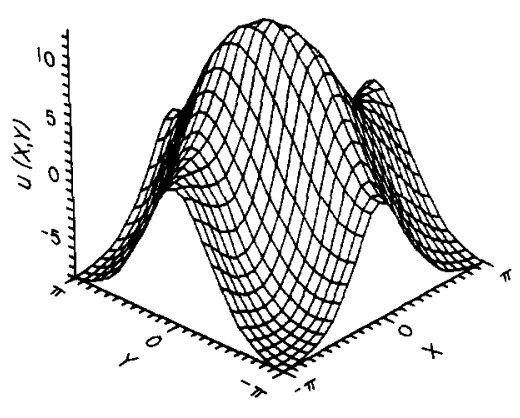

Fig. 8. Shape of the surface $u(X, Y)$ of the double cnoidal wave of the $\mathrm{KdV}$ for $a=5, b=1 .\left(X=x-c_{1} t, Y=k_{2}(x-\right.$ $c_{2} t$.

the sum of the cosine waves (the higher order terms of the Stokes expansion become more important). The other two points relate to the symmetry. For both plots, a $\mathrm{C}_{2}$ symmetry is evident $(X \rightarrow-X$ and $Y \rightarrow-Y$ is an identity operation). For the smaller-amplitude case, the contours show a near-reflection about the $X$ and $Y$ axes, showing the interaction of nearly linear cosine waves. As the amplitude increases, the shape changes in several ways. First, the peaks become narrower and troughs broader. This is merely the transition from the near-linear sine wave to the highly nonlinear solitary wave regime. Secondly, the contours of the wave crests are sloped. This is because the computed phase speeds, $c_{1}$ and $c_{2}$, differ from the solitary wave phase speeds, $c_{1}^{\text {sol }}$ and $c_{2}^{\text {sol }}$, by the phase shift divided by the time between collisions (see eq. (1.4)). As pointed out by Boyd [12], $c_{1}$ and $c_{2}$ are average speeds. In the figures, the slopes inside the central interaction region are opposite those outside of it: the phase shift makes up for the difference between the average and soliton speeds. This is confirmed by the $X-t$ plots,

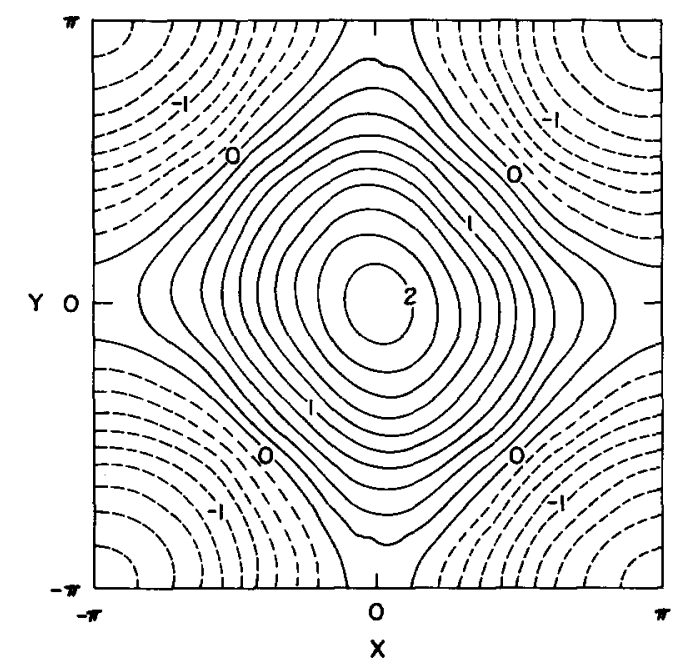

Fig. 9. Phase contours of $u(X, Y)$ for $a=1, b=1$. Note the strong apparent symmetries for this sinusoidal-like wave.

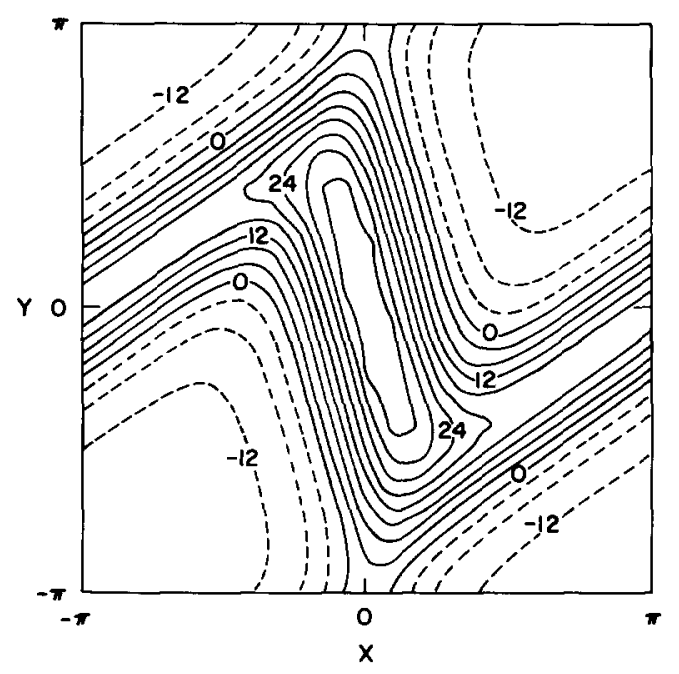

Fig. 10. Phase contours of $u(X, Y)$ for the $\mathrm{KdV}$ double cnoidal wave for $a=10, b=1$. Although the $\mathrm{C}_{2}$ symmetry is still evident, the lack of reflection about the $X$ and $Y$ axes indicates that this case is well into the solitary wave regime. 


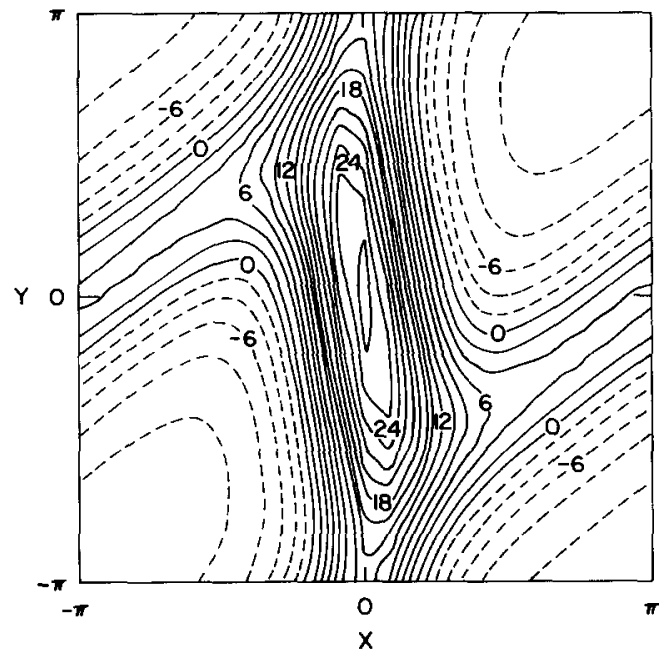

Fig. 11. Phase contours of $u(X, Y)$ for a double cnoidal wave of the KdV for $a=10, b=5$. The smaller value of $b$ causes an elongation along the $Y$-axis.

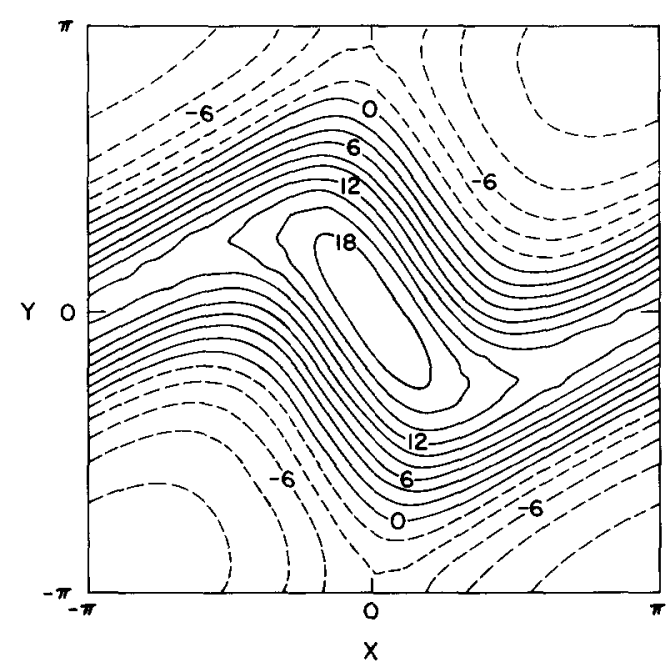

Fig. 12. Phase contours of $u(X, Y)$ for a double cnoidal wave of the $\mathrm{KdV}$ for $a=5, b=2$. While the wave in the $Y$ variable is the same height as in fig. 10, the $X$ wave is half of that, resulting in an elongation along the $X$-axis.

figs. 2 and 3, from which we may determine the direction of the adjustment. It is obvious that the tallest wave travels slower than the computed speed, but that it is phase shifted forward upon collision to compensate.

Figs. 11 and 12, when compared with Fig. 10, show the effect of modifying the amplitude ratio, $b$. Each of the three plots has an amplitude of either $\cos (X)$ or $\cos (Y)$ of 10 . In fig. 11, the $\cos (X)$ wave is twice the height of the $\cos (Y)$ component and the interaction area is elongated along the $Y$-axis with a steeper slope along the $X$ direction. The opposite is true in fig. 12, where $a=5$ and $b=2$ - the slope along the $Y$-axis is roughly 4 times that along the $X$-axis (versus a ratio of $2: 1$ when $a=10$ and $b=1$ ).

\subsection{Solitons versus sine waves and higher wavenumber waves}

Boyd [14] used arguments involving the modular transform of theta functions to show that the Fourier (near-linear) [1,2] mode is equivalent to the Gaussian (soliton-like) mode of $\{1,1\}$, where the square brackets denote the $k_{1}, k_{2}$ wavenumber pair in Fourier notation and the curly brackets are for Gaussian wavenumbers. We can see the same principle in fig. 1 . The numerical solution for this figure (denoted by the circles) was computed as a Fourier series with the highest-order cosine waves having wavenumbers 1 and 2. The exact solution for comparison was computed using two sech $^{2}$ soliton forms on each period (see (3.9)), which is equivalent to two Gaussian waves, each with a wavenumber of 1 . At small amplitude, the double cnoidal wave solution is roughly $u=a[\cos (X)+b \cos (Y)]$, where $X$ and $Y$ include the wavenumbers $k_{1}$ and $k_{2}$ (eq. (1.2)). Therefore, the solution of $[1,2]$ is the superposition of a wavenumber 1 and wavenumber 2 cosine waves: the result is one tall crest at the origin and a smaller crest at $x=\pi$, repeated periodically. At higher amplitude, these two peaks are solitons of different sizes - equivalent to the $\{1,1\}$ Gaussian representation. Thus, this simple boundary value approach reproduces the "paradox of wavenumbers" of more complicated methods [14]. 


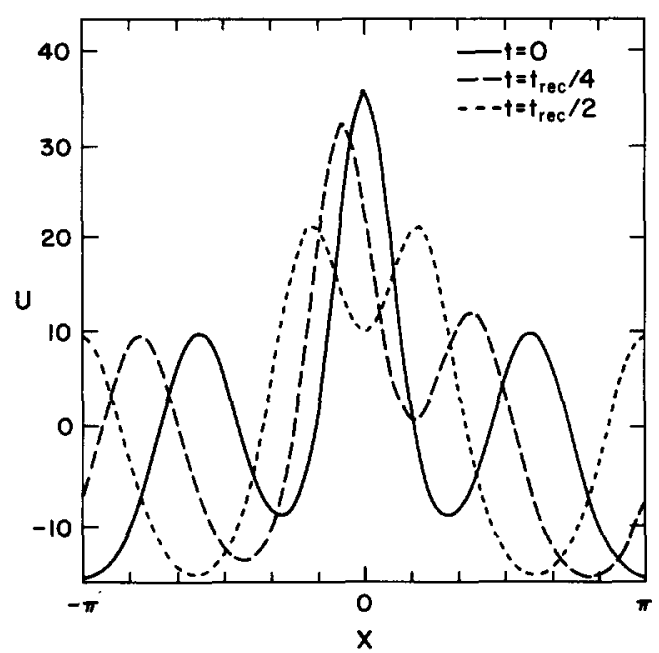

Fig. 13. Time evolution of the $\mathrm{KdV}$ double cnoidal wave with $k=3, a=10, b=1$.

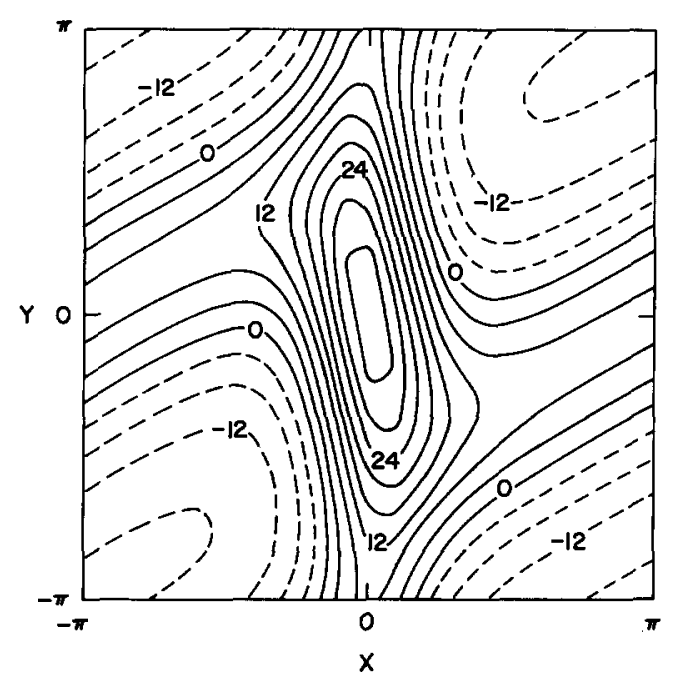

Fig. 14. Phase contours of $u(X, Y)$ for the $\mathrm{KdV}$ double cnoidal wave with $k=3, a=10, b=1$. $\left(X=x-c_{1} t, Y=\right.$ $\left.3\left(x-c_{2} t\right)\right)$.

Although we have concentrated on the $[1,2]$ double cnoidal wave, it is just as easy to compute the form for other pairs of wavenumbers. As an example, the wave with $k_{1}=1, k_{2}=3$ is studied here. Its basic form is shown at three different times in fig. 13. At time $t=0$, the profile consists of one large and two smaller waves: superposition of $\cos (x)$ with $\cos (3 x)$ produces one large wave at $x=0$ and two smaller ones at about $x= \pm 2 \pi / 3$. Thus we have $[1,3] \equiv\{1,2\}$. At time $\frac{1}{2} t_{\text {rec }}$, the large wave collides with one of the smaller waves. It is evident that this case is, in Lax's terminology [29], an example of the two solitons exchanging roles. A contour plot in the $X-Y$ plane is shown in fig. 14, which is similar in appearance to the $k_{2}=2$ case of fig. 10 . The difference is that the same pattern in fig. 14 would need to be repeated three times in the $Y$ direction for a complete mapping onto the $x-t$ plane. The plot in fig. 10, with $k_{2}=2$, requires only two periods in $Y$ to represent a complete mapping.

\section{Conclusions}

We have shown here that the boundary value approach to the $\mathrm{KdV}$ double cnoidal wave problem is a feasible solution method for analytical and numerical study. We can express the solution as $u(X, Y)$ where $X=k_{1}\left(x-c_{1} t\right)$ and $Y=k_{2}\left(x-c_{2} t\right)$ via a two-dimensional Fourier series in $X$ and $Y$. For small-amplitude waves, the coefficients of the Fourier series and the phase speed corrections are computed via a Stokes (or "Poincaré-Lindstedt") perturbation series. This solution is then extended to higher amplitude numerically using a Newton-Kantorovich iteration. The Fourier solutions thus provided are simple in form and may be used to study the behavior of the waves. All of the features of the time evolution studies appear, including the phase shifts upon collision. In the large-amplitude limit, the double cnoidal wave is a periodic repetition of the double soliton. The computed form agrees well with superposing two $\operatorname{sech}^{2}$ forms. The three separate types of soliton interactions described by Lax [29] were identifiable for finite-amplitude double cnoidal waves. Additionally, it is easy to replicate the results of 
more complicated methods [4, 12-14]: (1) the phase plots shown here demonstrate that the computed average phase speed is not equivalent to the instantaneous phase speeds outside of the interaction area-the average phase speed incorporates the effect of the collisional phase shift, and (2) the finite-amplitude double cnoidal wave is equivalently represented as a Fourier series based on two separate wavenumbers or as superimposed solitary waves.

In conclusion, the boundary value approach has proven successful in solving a nonlinear time evolution model of a physical phenomenon. The technique is not limited to small-amplitude solutions. The real power of this method lies in its applicability to problems for which no exact solution exists. Preliminary work has shown modest success in modeling the nonintegrable regularized long-wave equation [20]. It provides a Fourier solution which is a useful form for fitting observed data. In addition, this technique may be used to solve more complex problems, such as nonlinearly coupled systems.

\section{Acknowledgement}

This research was supported by the National Science Foundation under grant OCE8509923.

\section{References}

[1] D.J. Korteweg and G. deVries, Philos. Mag. Ser. 5, 39 (1895) 422.

[2] J.P. Boyd, Adv. Appl. Mech 27 (1989), in press.

[3] J.P. Boyd and S.E. Haupt, Nonlinear Ocean Physics: Proceedings of the Enrico Fermi Summer School, 1989, ed. A.R. Osborne (North-Holland, Amsterdam), in press.

[4] J.P. Boyd, J. Math. Phys. 23 (1982) 375.

[5] M.J. Ablowitz and H. Segur, Solitons and the Inverse Scattering Transform (Soc. Industr. Appl. Math., Philadelphia, PA 1981) $425 \mathrm{pp}$.

[6] S.P. Novikov, Funct. Anal. Appl. 8 (1974) 236.

[7] P.D. Lax, Commun. Pure Appl. Math. 28 (1975) 141.

[8] B.A. Dubrovin, V.B. Matseev and S.P. Novikov, Russian Math. Surv. 31 (1976) 59.

[9] A.R. Osborne and L. Bergamasco, Nuovo Cimento 85B (1985) 229.

[10] A.R. Osborne and L. Bergamasco, Physica D 21 (1986) 26.

[11] A. Nakamura, J. Phys. Soc. Japan 47 (1979) 1701.

[12] J.P. Boyd, J. Math. Phys. 25 (1984) 3390.

[13] J.P. Boyd, J. Math. Phys. 25 (1984) 3402.

[14] J.P. Boyd, J. Math. Phys. 25 (1984) 3415.

[15] H. Segur and A. Finkel, Stud. Appl. Math. 73 (1985) 183.

[16] B.B. Kadomtsev and V.I. Petviashvili, Sov. Phys. Dokl. 15 (1970) 539.

[17] T.B. Benjamin, J.L. Bona, and J.J. Mahony, Phil. Trans. Roy. Soc. A 272 (1972) 47.

[18] S. Pierini, J. Phys. Oceanog. 19 (1989) 755

[19] J. Hammack, N. Scheffner and H. Segur, Nonlinear Ocean Physics: Proceedings of the Enrico Fermi Summer School, 1989, ed. A.R. Osborne (North-Holland, Amsterdam), in press.

[20] S.E. Haupt, Solving nonlinear wave problems with spectral boundary value techniques, $\mathrm{Ph} . \mathrm{D}$. Dissertation, The University of Michigan (1988) $157 \mathrm{pp}$.

[21] J.P. Boyd, Physica D 21 (1986) 227.

[22] G.B. Whitham, Linear and Nonlinear Waves (Wiley, New York, 1974).

[23] A.H. Nayfeh, Perturbation Methods (Wiley, New York, 1973) 425 pp.

[24] S.E. Haupt and J.P. Boyd, Wave Motion 10 (1988) 83.

[25] L.V. Kantorovich, Dokl. Akad. Nauk SSSR 59 (1948) 1237.

[26] H.J. Norton, Computer J. 7 (1964) 76.

[27] S.A. Orszag, Stud. Appl. Math. 50 (1971) 293.

[28] R.E. Bank and T.F. Chan, SIAM J. Sci. Stat. Comput. 7 (1986) 540.

[29] P.D. Lax, Commun. Pure Appl. Math. 21 (1968) 467. 